\title{
Radiomics-Based Pretherapeutic Prediction of Non-response to Neoadjuvant Therapy in Locally Advanced Rectal Cancer
}

\author{
Xuezhi Zhou, $\mathrm{PhD}^{1,3}$, Yongju Yi, $\mathrm{PhD}^{2}$, Zhenyu Liu, $\mathrm{PhD}^{3,7}$, Wuteng Cao, MD ${ }^{4}$, Bingjia Lai, $\mathrm{MD}^{5}$, \\ Kai Sun, $\mathrm{PhD}^{1}$, Longfei Li, $\mathrm{PhD}^{6}$, Zhiyang $\mathrm{Zhou}, \mathrm{MD}^{4}$, Yanqiu Feng, $\mathrm{PhD}^{2}$, and Jie Tian, $\mathrm{PhD}^{1,3,7,8}$ \\ ${ }^{1}$ Engineering Research Center of Molecular and Neuro Imaging of Ministry of Education, School of Life Science and \\ Technology, Xidian University, Xi'an, Shaanxi, China; ${ }^{2}$ Guangdong Provincial Key Laboratory of Medical Image \\ Processing, School of Biomedical Engineering, Southern Medical University, Guangzhou, China; ${ }^{3}$ CAS Key Laboratory \\ of Molecular Imaging, Institute of Automation, Chinese Academy of Science, Beijing, China; ${ }^{4}$ Department of Radiology, \\ The Sixth Affiliated Hospital, Sun Yat-Sen University, Guangzhou, China; ${ }^{5}$ Department of Radiology, Sun Yat-Sen \\ Memorial Hospital, Sun Yat-Sen University, Guangzhou, China; ${ }^{6}$ Collaborative Innovation Center for Internet Healthcare, \\ Zhengzhou University, Zhengzhou, Henan, China; ${ }^{7}$ University of Chinese Academy of Science, Beijing, China; ${ }^{8}$ Beijing \\ Advanced Innovation Center for Big Data-Based Precision Medicine, Beihang University, Beijing, China
}

\begin{abstract}
Objective. The aim of this study was to investigate whether pretherapeutic, multiparametric magnetic resonance imaging (MRI) radiomic features can be used for predicting non-response to neoadjuvant therapy in patients with locally advanced rectal cancer (LARC).

Methods. We retrospectively enrolled 425 patients with LARC [allocated in a 3:1 ratio to a primary $(n=318)$ or validation $(n=107)$ cohort] who received neoadjuvant therapy before surgery. All patients underwent T1-weighted, T2-weighted, diffusion-weighted, and contrastenhanced T1-weighted MRI scans before receiving neoadjuvant therapy. We extracted 2424 radiomic features from the pretherapeutic, multiparametric MR images of each patient. The Wilcoxon rank-sum test, Spearman
\end{abstract}

Electronic supplementary material The online version of this article (https://doi.org/10.1245/s10434-019-07300-3) contains supplementary material, which is available to authorized users.

Xuezhi Zhou, Yongju Yi, Zhenyu Liu, and Wuteng Cao contributed equally to this work, and should be considered as co-first authors.

(C) The Author(s) 2019

First Received: 19 November 2018;

Published Online: 18 March 2019

Y. Feng, PhD

e-mail: foree@163.com

J. Tian, $\mathrm{PhD}$

e-mail: jie.tian@ia.ac.cn correlation analysis, and least absolute shrinkage and selection operator regression were successively performed for feature selection, whereupon a multiparametric MRIbased radiomic model was established by means of multivariate logistic regression analysis. This feature selection and multivariate logistic regression analysis was also performed on all single-modality MRI data to establish four single-modality radiomic models. The performance of the five radiomic models was evaluated by receiver operating characteristic (ROC) curve analysis in both cohorts.

Results. The multiparametric, MRI-based radiomic model based on 16 features showed good predictive performance in both the primary $(p<0.01)$ and validation $(p<0.05)$ cohorts, and performed better than all single-modality models. The area under the ROC curve of this multiparametric MRI-based radiomic model achieved a score of 0.822 (95\% CI 0.752-0.891).

Conclusions. We demonstrated that pretherapeutic, multiparametric MRI radiomic features have potential in predicting non-response to neoadjuvant therapy in patients with LARC.

The standard treatment for patients with locally advanced rectal cancer (LARC) is neoadjuvant chemoradiotherapy followed by total mesorectal excision. ${ }^{1}$ Many studies have demonstrated that the response to neoadjuvant therapy affects prognosis, and that, in particular, pathologic complete response $(\mathrm{pCR})$ has a favorable effect on outcome. $^{2-4}$ 
However, a sizeable proportion of patients show nonresponse to neoadjuvant therapy. These non-responders can be defined as those who have no tumor regression changes, based on resection specimens, after neoadjuvant therapy. A number of studies have reported that approximately $7 \%$ of LARC patients showed non-response to preoperative chemoradiotherapy and more than $20 \%$ developed grade 3-4 toxic effects such as diarrhea, nausea, hematological infection, and fever. ${ }^{5-7}$ These non-responders may benefit little from neoadjuvant therapy, but still experience the related toxicity. More importantly, tumor progression may occur in a portion of these patients during therapy. Thus, accurately predicting non-responders before administering neoadjuvant therapy is important for devising a personalized treatment plan, which includes avoiding overtreatment and choosing alternative treatments in a timely manner. However, there is currently no robust method for accurately stratifying patients into non-response and response groups, other than by pathologic evaluation after completing neoadjuvant therapy.

Due to the heterogeneity of tumors, ${ }^{8}$ accurately predicting a non-response to neoadjuvant therapy remains challenging. Numerous studies have sought molecular biomarkers for early identification of good and poor responders to neoadjuvant therapy in patients with LARC, but no robust predictive factors have been identified. ${ }^{9-12}$

Magnetic resonance imaging (MRI) is the most commonly used diagnostic imaging approach for rectal tumors. Pretherapeutic MRI features, such as tumor volume, tumor height, depth of tumor penetration, and absence of extramural venous invasion, have been associated with a better response to therapy; ${ }^{13-15}$ however, these visual features have a poor ability to accurately predict non-response for administering therapy.

Radiomics, a novel approach to medical image analysis, can extract high-throughput quantitative features from images, and thus provides a wealth of information that cannot be assessed visually but is associated with tumoral heterogeneity. ${ }^{16}$ Therefore, tumor characteristics can be better understood by analyzing radiomic features. Radiomics has facilitated progress in precise diagnosis, ${ }^{17,18}$ prediction of lymph node metastasis, ${ }^{19,20}$ and survival analysis. $^{21,22}$ Previous radiomic studies focused on therapeutic responses in LARC patients were mainly based on combining pre- and post-therapeutic imaging data or images obtained during neoadjuvant therapy. ${ }^{23-25}$ Most of these studies focused on single-modality imaging, such as dynamic contrast-enhanced MRI, ${ }^{26}$ diffusion-weighted MRI, ${ }^{27}$ T2-weighted MRI, ${ }^{28}$ and fluorodeoxyglucose positron emission tomography, ${ }^{29}$ which may have inherent limitations in reflecting tumor biology. A recent study with a small sample size demonstrated that features derived from multiparametric MRI performed better than that derived from single-modality MRI in terms of predicting good responders from poor responders, but the study lacked independent validation. ${ }^{30}$

Based on the initial success of MRI-based radiomics in assessing pCR, we hypothesized that pretherapeutic MRIbased radiomics would have potential in predicting nonresponders to neoadjuvant therapy, and that multiparametric MRI would be more effective than single-modality MRI in this task. Therefore, in this study we investigated whether a pretherapeutic, multiparametric MRI-based radiomic model could predict non-responders to neoadjuvant therapy, as integrating a radiomic model and clinical practice may facilitate a flexible therapeutic schedule prior to commencing neoadjuvant therapy.

\section{MATERIALS AND METHODS}

\section{Patients}

This retrospective single-center study was approved by the Ethics Committee of the Sixth Affiliated Hospital, Sun Yat-sen University. The need for informed patient consent was waived due to the retrospective nature of this study. Overall, 425 patients with biopsy-proven LARC receiving pretherapeutic, multiparametric MRI examination and standard treatment between November 2012 and May 2017 at this hospital were enrolled; 248 of these patients underwent 5-fluorouracil, leucovorin, and oxaliplatin treatment, and 177 underwent 5-fluorouracil and concurrent radiation treatment (total dose 46.0-50 Gy). The multiparametric MRI consisted of T1-weighted fast spin echo imaging $(\mathrm{T} 1 \mathrm{w}), \mathrm{T} 2$-weighted fast spin echo imaging (T2w), diffusion-weighted imaging (DWI), and contrastenhanced T1-weighted fast spin echo imaging (CE-T1w). Two gastrointestinal radiologists with 10 and 30 years' experience reassessed the rectal tumor in MRI without medical record information, and obtained consistent staging results, including clinical $\mathrm{T}$ stage (cT stage) and clinical $\mathrm{N}$ stage ( $\mathrm{cN}$ stage). Other clinical information, including sex, age, and carcinoembryonic antigen (CEA; cutoff $\geq 5 \mathrm{ng} / \mathrm{ml}, \quad<5 \mathrm{ng} / \mathrm{ml}$ ) blood level ${ }^{31}$ was completely recorded. Appendix 1 describes the recruitment of patients.

Pathologic response after neoadjuvant therapy was reassessed based on resection specimens by two patholo- 
gists with 10 and 20 years' experience in consensus. Pathologic grading of primary tumor regression was performed according to the four-tier American Joint Committee on Cancer (AJCC) tumor regression grade (TRG) system. ${ }^{32}$ The four TRG groups are: TRG 0 , no residual tumor cells; TRG 1, single tumor cell or small group of tumor cells; TRG 2, residual cancer with desmoplastic response; and TRG 3, minimal evidence of tumor response. According to the AJCC TRG system, patients were categorized into two groups: non-responders (TRG 3) and responders (TRG 0-2). Patients were then randomly divided into two independent cohorts: a primary cohort used for modeling (proportion $=3 / 4$; TRG 3: $n=39$, TRG 0-2: $n=279$ ) and a validation cohort used for testing (proportion $=1 / 4$; TRG 3: $n=13$, TRG 0-2: $n=94)$. The clinical characteristics of patients in the primary and validation cohorts are summarized in Table 1.

\section{Image Data Acquisition and Radiomic Signature Construction}

Appendix 2 describes the pretherapeutic, multiparametric MRI acquisition; Appendix 3 describes details of tumor masking and radiomic feature extraction; and
Appendix 4 describes details of the feature selection method and radiomic signature construction. It should be noted that we built a multiparametric MRI-based radiomic (MPR) model and four single-modality models via the same workflow on the primary cohort only.

\section{Evaluation and Comparison of Different Prediction Models}

Receiver operating characteristic (ROC) curve analysis was conducted in both cohorts to evaluate the predictive ability of radiomic signatures. The Delong test was performed to estimate whether the difference between two arbitrary ROC curves was statistically significant.

Univariate and multivariate logistic regression analyses were conducted to select the most useful predictive clinical variables (Wald test $p$-values $<0.05$ ). A multivariate logistic prediction model was then built by combining the MPR signature and the selected clinical variables; this was termed the CMPR model.

Calibration curves accompanied by the Hosmer-Lemeshow goodness-of-fit (GOF) test were plotted to assess the consistency between the estimated probability and the actual rate of TRG 3; $p$ values $>0.05$ indicate a good fit of
TABLE 1 Clinical characteristics of patients in the primary and validation cohorts

\begin{tabular}{|c|c|c|c|c|c|c|}
\hline \multirow[t]{2}{*}{ Characteristic } & \multicolumn{2}{|l|}{ Primary cohort } & \multirow[t]{2}{*}{$p$ value } & \multicolumn{2}{|c|}{ Validation cohort } & \multirow[t]{2}{*}{$p$ value } \\
\hline & TRG 3 & TRG $0-2$ & & TRG 3 & TRG $0-2$ & \\
\hline Age, years & $50.31 \pm 13.89$ & $53.98 \pm 12.39$ & 0.089 & $57.46 \pm 8.99$ & $54.06 \pm 11.11$ & 0.294 \\
\hline Sex & & & 0.194 & & & 0.509 \\
\hline Male & 31 & 191 & & 11 & 69 & \\
\hline Female & 8 & 88 & & 2 & 25 & \\
\hline CEA & & & 0.384 & & & 0.387 \\
\hline Positive & 18 & 107 & & 4 & 42 & \\
\hline Negative & 21 & 172 & & 9 & 52 & \\
\hline cT stage & & & 1 & & & 0.744 \\
\hline $\mathrm{T} 2$ & 3 & 22 & & 0 & 4 & \\
\hline $\mathrm{T} 3$ & 29 & 203 & & 11 & 71 & \\
\hline $\mathrm{T} 4$ & 7 & 54 & & 2 & 19 & \\
\hline $\mathrm{cN}$ stage & & & 0.573 & & & 0.196 \\
\hline No & 6 & 62 & & 4 & 21 & \\
\hline N1 & 18 & 108 & & 3 & 47 & \\
\hline $\mathrm{N} 2$ & 15 & 109 & & 6 & 26 & \\
\hline
\end{tabular}

Continuous data are expressed as mean \pm SD. $p$ values for categorical variables, such as sex and CEA, were from the Fisher's exact test analysis. $p$ values for categorical variables, such as sex and CEA, were from the Fisher's exact test analysis. $p$ values for categorical variables, such as $\mathrm{cT}$ stage and $\mathrm{cN}$ stage, were from the Pearson's Chi square test analysis. $p$ values for continuous variables were from the independent samples $t$ test analysis

$T R G$ tumor regression grade, $C E A$ carcinoembryonic antigen, $c T$ stage clinical T stage, $c N$ stage clinical $\mathrm{N}$ stage 
the model. ${ }^{33}$ The classification accuracy, sensitivity, and specificity, according to the Youden index cut-off, ${ }^{34}$ were calculated to quantize the discrimination ability of the prediction models in both cohorts.

\section{RESULTS}

\section{Demographic and Clinical Characteristics}

Detailed information regarding study participants is presented in Table 1 and Appendix Table A3. In this study, we enrolled 425 LARC patients who underwent standard neoadjuvant therapy, including $52(12.23 \%)$ non-responders (42 males, 10 females) and $373(87.77 \%)$ responders (260 males, 113 females). There were no significant differences in clinical variables between cohorts.
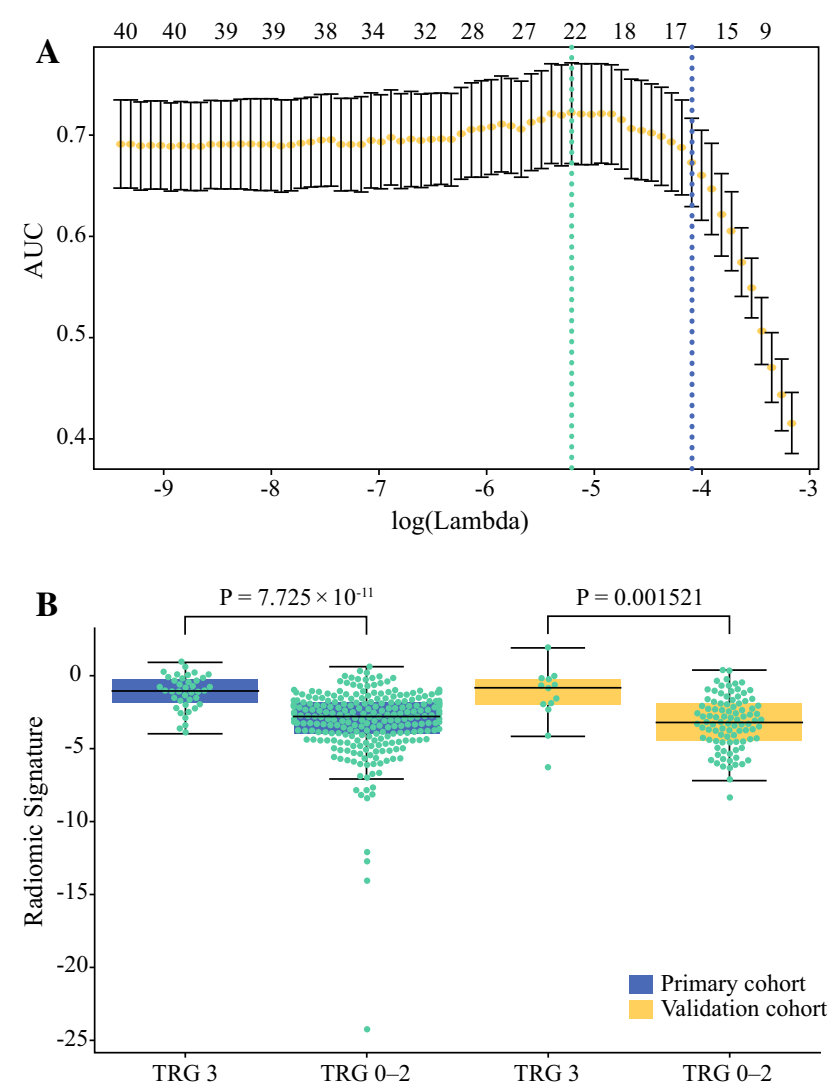

FIG. 1 Feature selection using LASSO and distribution of the MPR signature. a The 'Lambda' parameter was chosen via 1 standard error of the minimum criterion, as marked by the blue dotted vertical line. b Distribution of the MPR signature in both cohorts. LASSO least absolute shrinkage and selection operator, $M R I$ magnetic resonance imaging, $M P R$ multiparametric MRI-based radiomic, $T R G$ tumor regression grade, $A U C$ area under the curve
Feature Selection and Radiomic Signature Construction

Sixteen features were selected for constructing the MPR model. In both cohorts, the MPR signature was significantly higher in the TRG 3 group than in the TRG 0-2 group, as shown in Fig. 1. Single-modality radiomic models, including the apparent diffusion coefficient (ADC; seven selected features), T1w (five selected features), CE$\mathrm{T} 1 \mathrm{w}$ (three selected features), and T2w (three selected features) models, were also constructed.

\section{Evaluation and Comparison of Different Prediction Models}

The MPR model yielded the highest area under the curve (AUC) in both cohorts, and was statistically significantly higher than other single-modality models $(p<0.05)$ in the primary cohort, as shown in Fig. 2.

In the multivariate analysis, both age [odds ratio (OR) 0.57, $p=0.0071]$ and MPR signature (OR 17.78, $p<0.001)$ were significantly associated with non-response, as shown in Table 2. Thus, the CMPR model was built using logistic regression, combining age and the MPR signature in the primary cohort.

In both cohorts, the CMPR model performed similarly as the MPR model (Delong test $p$ values $>0.05$ ) in the ROC analysis. However, the calibration curve generated from the CMPR model did not show good consistency between prediction and actual observation in the validation cohort (Hosmer-Lemeshow GOF test $p$ value $<0.05$ ), as shown in Fig. 3. The AUC, accuracy, sensitivity, and specificity of the MPR and CMPR models, according to the Youden index cut-off, are listed in Appendix Table A4.

\section{DISCUSSION}

Our study demonstrated that pretherapeutic, multiparametric MRI radiomic features have great potential in predicting non-responders to neoadjuvant therapy in patients with LARC. Based on the 16 most predictive features, as listed in Appendix Table A5, we established the MPR model by multivariate logistic regression analysis. In an independent validation cohort, the MPR model showed good predictive performance (AUC 0.773).

For radiomic feature extraction, we mainly used the Laplacian of Gaussian (LoG) filter for image preprocessing. An LoG filter can smooth images through different parameter scale settings, which may help to decrease the influence of noise. It can also enhance textural details, 
A

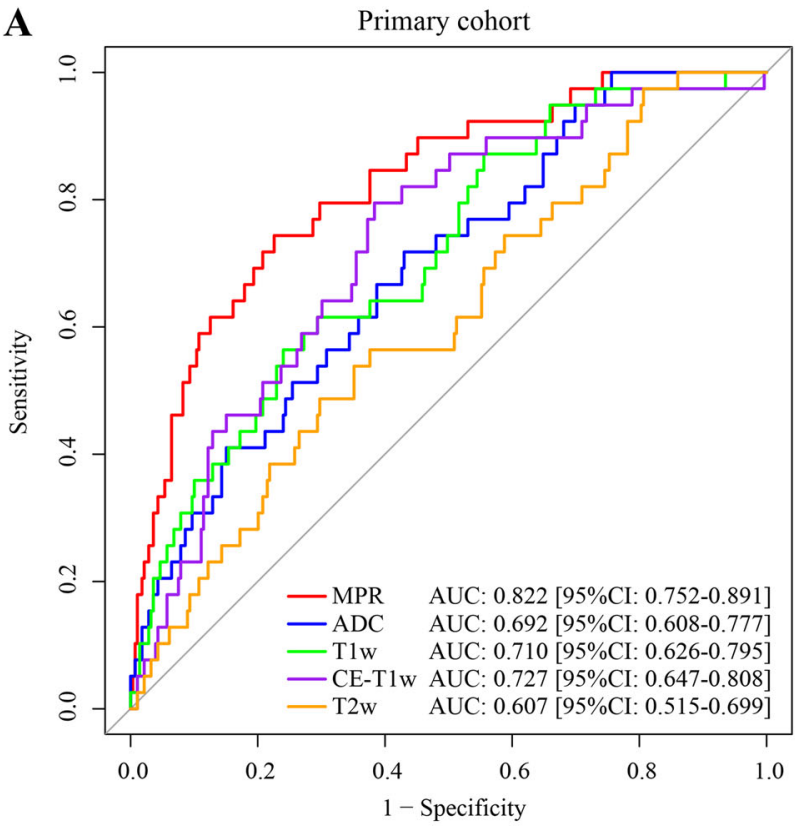

C

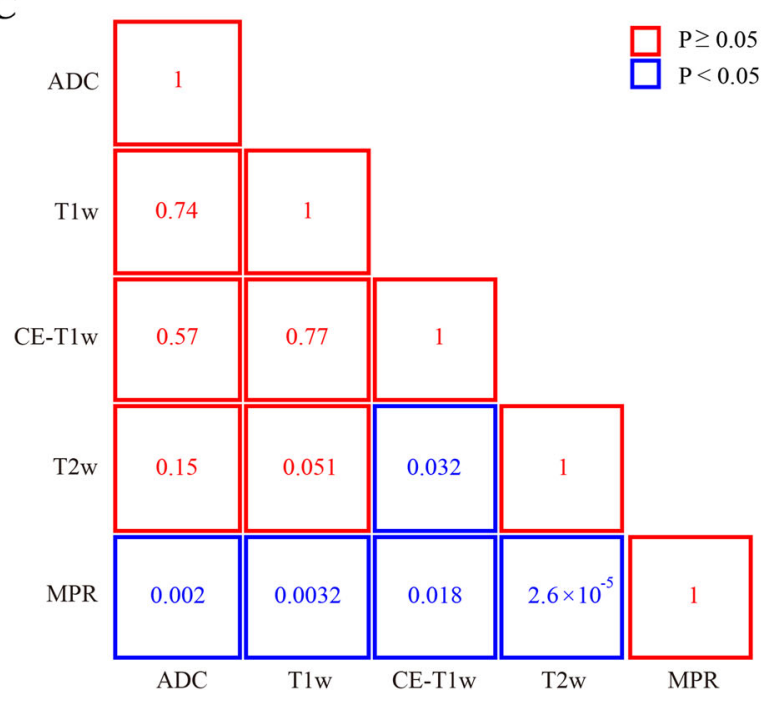

FIG. 2 Performance comparison of the MPR and single-modality models. a, b ROC curves. c, $\mathbf{d}$ Delong test $p$-values. $R O C$ receiver operating characteristic, $A U C$ area under the curve, $M P R$ multiparametric magnetic resonance imaging-based radiomic model,

which will help to increase the efficiency of capturing phenotypic features that map to tumoral heterogeneity. Because of these advantages, LoG filters have been wildly used in radiomic studies, mostly using CT images. ${ }^{19,35,36}$ In an early MRI-based radiomic study, an LoG filter was used to extract features for predicting $\mathrm{pCR}$ in rectal cancer patients, ${ }^{37}$ while, in the present study, we extracted LoG features on five scales, in addition to the features from the original images. We then selected 16 features and linearly constructed an MPR signature by logistic regression analysis. In the MPR model, there was an apparent difference
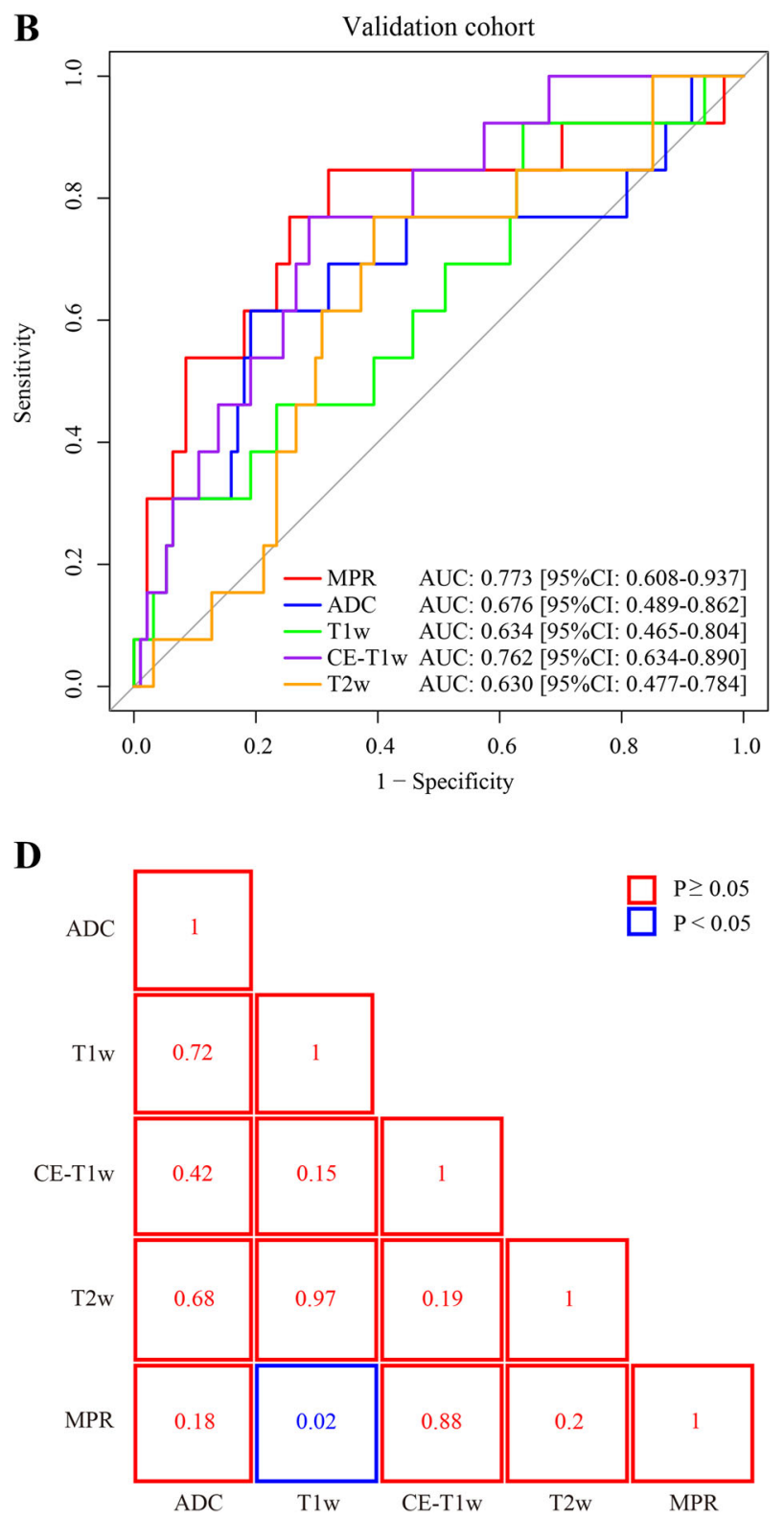

$A D C$ apparent diffusion coefficient, T1w T1-weighted, CE-T1w contrast-enhanced T1-weighted, T2w T2-weighted, $C I$ confidence interval

between the number of features generated from LoG filters and that from the original images. Given that the LoG filter features accounted for a higher proportion of the features employed in the MPR model (10/16) than the original image features, it appears that LoG features may be more suitable than original image features for predicting a nonresponse. Nevertheless, original image features remain indispensable to the MPR model because the odds ratio of the feature GLCM_entropy_135 generated from the original image was the largest among all the features employed (OR 3.2808, $p=0.0059$ ). 
TABLE 2 Univariate and multivariate logistic regression analyses for clinical characteristics and MPR signature

\begin{tabular}{|c|c|c|c|c|c|c|}
\hline \multirow[t]{2}{*}{ Parameter } & \multicolumn{3}{|l|}{ Univariate } & \multicolumn{3}{|c|}{ Multivariate } \\
\hline & $p$ value & OR & $95 \% \mathrm{CI}$ & $p$ value & OR & $95 \% \mathrm{CI}$ \\
\hline Sex & 0.1645 & 1.78 & $0.78-4.04$ & 0.8063 & 1.21 & $0.45-2.81$ \\
\hline Age & 0.0903 & 0.74 & $0.52-1.05$ & 0.0055 & 0.55 & $0.36-0.84$ \\
\hline CEA & 0.3515 & 1.38 & $0.70-2.70$ & 0.4292 & 1.37 & $0.86-1.10$ \\
\hline cT stage & 0.4768 & 0.63 & $0.17-2.27$ & 0.2003 & 0.41 & $0.63-3.01$ \\
\hline $\mathrm{cN}$ stage & 0.6303 & 1.24 & $0.50-3.05$ & 0.6935 & 0.80 & $0.27-2.38$ \\
\hline MPR signature & $<0.0001$ & 14.51 & $5.82-36.13$ & $<\mathbf{0 . 0 0 0 1}$ & 17.78 & $6.73-46.99$ \\
\hline
\end{tabular}

The $p$ value was from the Wald test analysis. Bold values indicate $p<0.05$

$O R$ odds ratio, $C I$ confidence interval, $M P R$ multiparametric magnetic resonance imaging-based radiomic model, $C E A$ carcinoembryonic antigen, $c T$ stage clinical $\mathrm{T}$ stage, $c N$ stage clinical $\mathrm{N}$ stage
In the present study, we also investigated differences in the performance of the MPR and single-modality models. The CE-T1w model was the best-performing singlemodality model in both the primary and validation cohorts. Compared with other imaging modalities, CE-T1w images enhanced the contrast of the tumor area, making the texture distribution clearer, which may have helped to improve the predictive ability of the textural feature. The MPR model performed significantly better than the CE-T1w model ( $p=0.01775)$ in the primary cohort, implying that each modality has limitations in presenting tumor characteristics. Fusing multimodal information could facilitate a more comprehensive description of tumor characteristics, and could undoubtedly help to establish a more precise prediction model.

Some rectal cancer studies have shown that incorporating clinical variables into the radiomics model can improve predictive performance. ${ }^{19,38}$ Hence, we attempted to combine the MPR signature with a clinically associated variable (age) to establish the CMPR model. However, compared with the MPR model, the CMPR model did not improve the predictive ability in the validation cohort. More specifically, the MPR and CMPR models achieved the same predictive accuracy (accuracy $=76.64 \%$ ), and, compared with the MPR model, the specificity of the CMPR model increased by only $0.97 \%$, while the sensitivity of the CMPR model decreased by $7.69 \%$. Predictive robustness evaluation also showed that the predictive performance of the two models was similar. Therefore, in the present study, we deemed that the MPR model was more suitable.

At present, our understanding of factors influencing the effect of chemoradiotherapy is extremely poor. Several factors, such as apoptosis, proliferation, hypoxia, and cell cycle, have been investigated in this respect. ${ }^{39,40}$ Gene mutations in the related pathways have been shown to cause resistance to chemoradiation. With the help of radiomics and increasing medical imaging data, it may be possible to discover a relationship between medical images and non-response. In future, mapping the predictive image features to various molecules will help to discover major biomarkers related to resistance to chemoradiation in a more efficient and economical way.

There are some limitations to this study. First, the proportion of non-responders was small in the cohort as a whole, and, second, this was a single-center study. Repeatability and reproducibility of radiomic features are mainly affected by two factors: segmentation and image acquisition protocol. ${ }^{41}$ In our study, the region of interest was manually delineated by two experienced gastrointestinal MRI radiologists in consensus, resulting in a more accurate segmentation and decreasing the influence derived from interobserver variability. In addition, all these patients were scanned by the same MRI machine with fixed scanning parameters, avoiding the potential impact of a heterogeneous acquisition protocol. In spite of this, and considering the large heterogeneity of data from different centers, the model should be validated using a multicenter dataset before being applied to other institutions. Third, additional clinical information should be collected in future to investigate whether the CMPR model could perform better than the MPR model.

\section{CONCLUSIONS}

This study showed that pretherapeutic, multiparametric MRI radiomic features have potential in predicting nonresponse to neoadjuvant therapy prior to commencing this 
$\mathbf{A}$

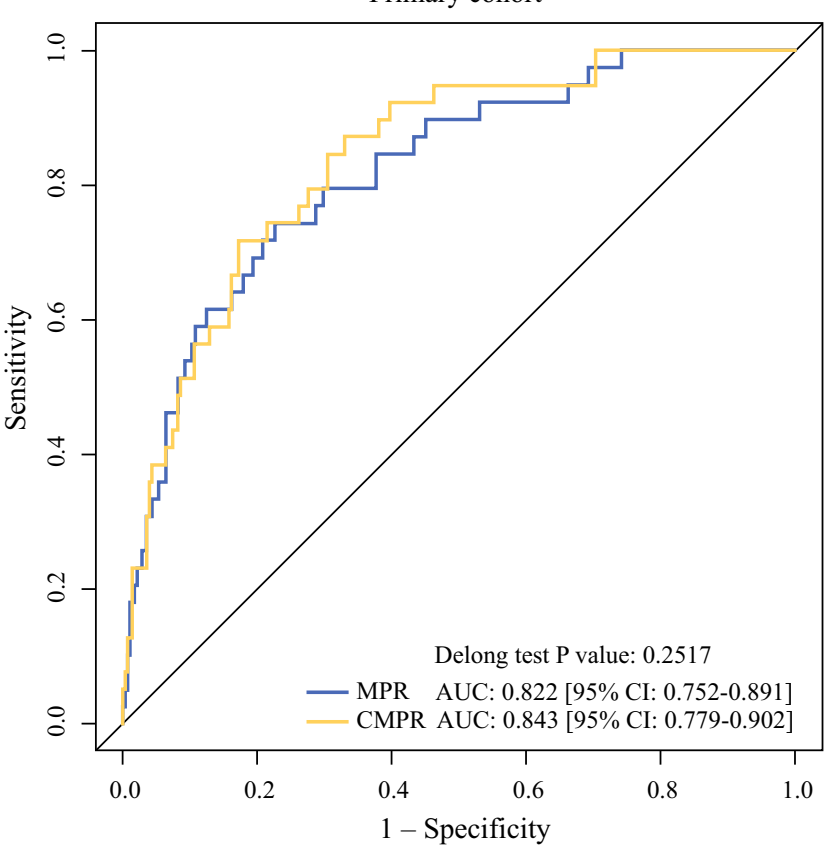

C

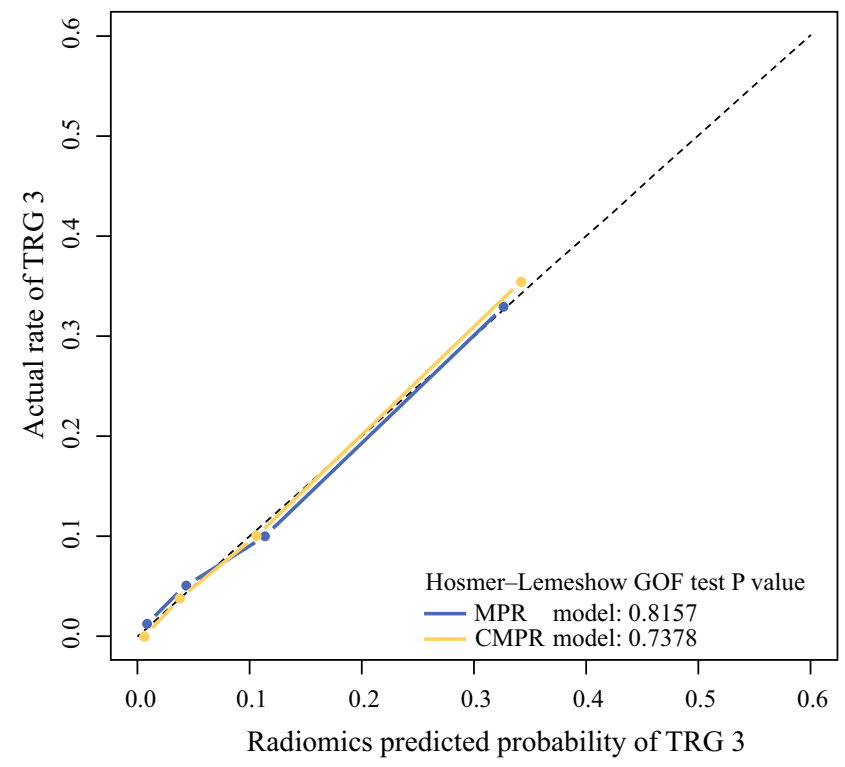

FIG. 3 Performance comparison of the MPR and CMPR models. a, b ROC curves. c, d Calibration curves. $R O C$ receiver operating characteristic, $A U C$ area under the curve, $M P R$ multiparametric

therapy. In addition, multiparametric MRI was more effective than single-modality MRI in this prediction task. This multiparametric MRI-based radiomic model may help doctors make more appropriate treatment plans for patients with LARC before they receive neoadjuvant therapy.

ACKNOWLEDGEMENTS This paper was supported by the Beijing Natural Science Foundation under Grant Number 7182109;

\section{B}

Validation cohort

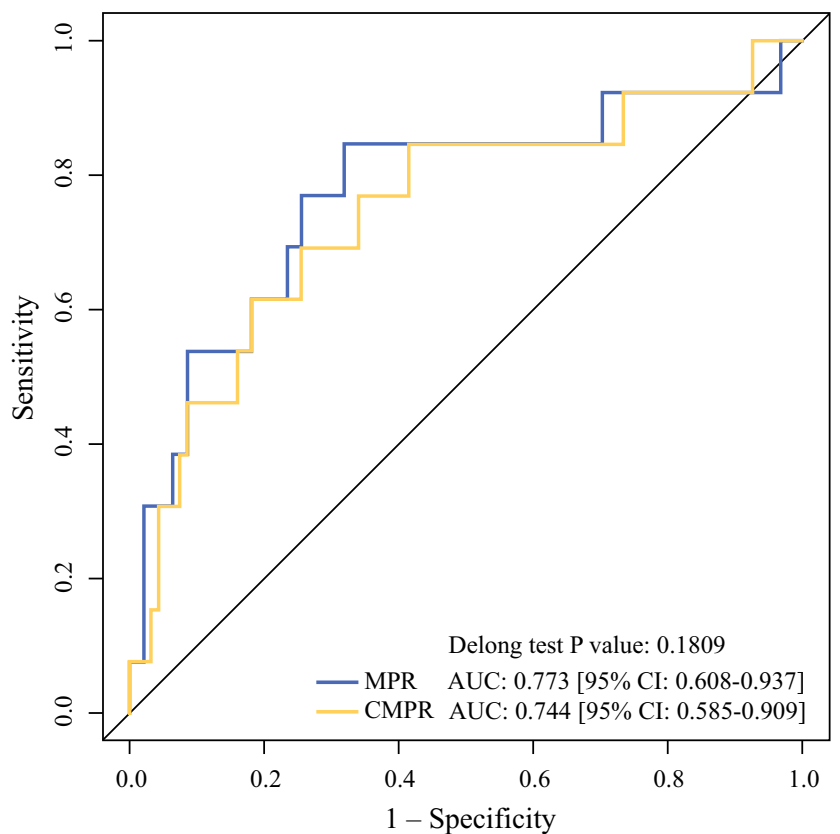

D

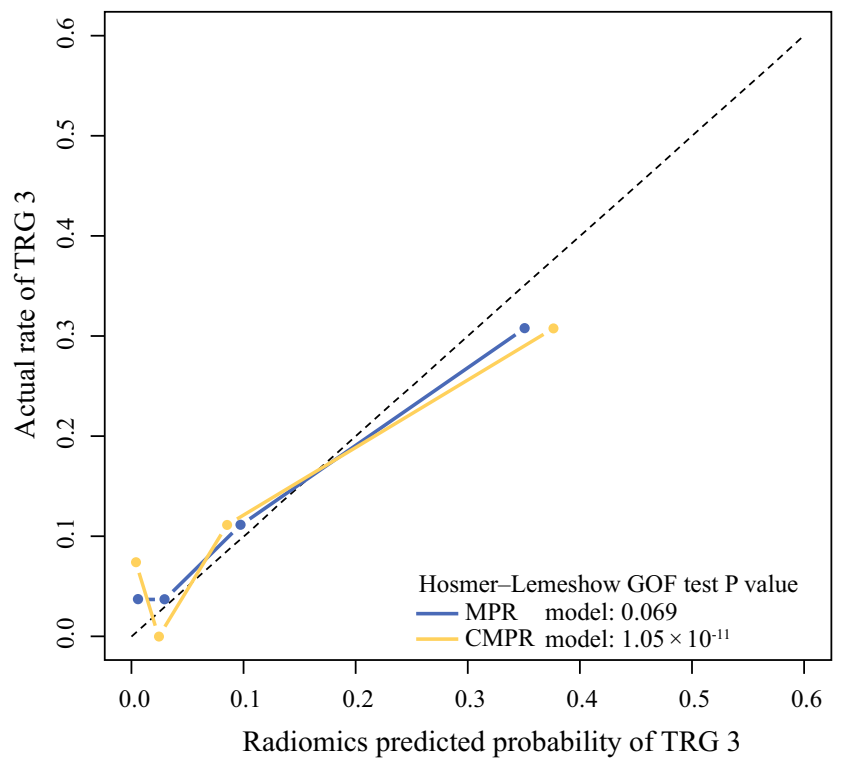

magnetic resonance imaging-based radiomic model, $C M P R$ combined MPR signature and age model, TRG tumor regression grade, $C I$ confidence interval, GOF goodness-of-fit

the National Natural Science Foundation of China under Grant Numbers 81772012, 81227901, and 81527805; the National Key Research and Development Plan of China under Grant Number 2017YFA0205200; and the Chinese Academy of Sciences under Grant Numbers GJJSTD20170004 and QYZDJ-SSW-JSC005. The authors would like to acknowledge the instrumental and technical support of the multimodal biomedical imaging experimental platform, Institute of Automation, Chinese Academy of Sciences. 
CONFLICT OF INTEREST Xuezhi Zhou, Yongju, Zhenyu Liu, Wuteng Cao, Bingjia Lai, Kai Sun, Longfei Li, Zhiyang Zhou, Yanqiu Feng, and Jie Tian declare that they have no conflicts of interest.

OPEN ACCESS This article is distributed under the terms of the Creative Commons Attribution 4.0 International License (http://crea tivecommons.org/licenses/by/4.0/), which permits unrestricted use, distribution, and reproduction in any medium, provided you give appropriate credit to the original author(s) and the source, provide a link to the Creative Commons license, and indicate if changes were made.

\section{REFERENCES}

1. Benson AB, Venook AP, Al-Hawary MM, et al. Rectal cancer, version 2.2018, NCCN clinical practice guidelines in oncology. $J$ Natl Compr Cancer Netw. 2018;16(7):874-901.

2. Park IJ, You YN, Agarwal A, et al. Neoadjuvant treatment response as an early response indicator for patients with rectal cancer. J Clin Oncol. 2012;30(15):1770.

3. Patel UB, Taylor F, Blomqvist L, et al. Magnetic resonance imaging-detected tumor response for locally advanced rectal cancer predicts survival outcomes: MERCURY experience. $J$ Clin Oncol. 2011;29(28):3753-3760.

4. Fokas E, Ströbel P, Fietkau R, et al. Tumor regression grading after preoperative chemoradiotherapy as a prognostic factor and individual-level surrogate for disease-free survival in rectal cancer. J Natl Cancer Inst. 2017;109(12):djx095.

5. Bosset JF, Collette L, Calais G, et al. Chemotherapy with preoperative radiotherapy in rectal cancer. $N$ Engl $J$ Med. 2006;355(11):1114-1123.

6. Roh MS, Colangelo LH, O'Connell MJ, et al. Preoperative multimodality therapy improves disease-free survival in patients with carcinoma of the rectum: NSABP R-03. J Clin Oncol. 2009;27(31):5124.

7. Rödel C, Liersch T, Becker H, et al. Preoperative chemoradiotherapy and postoperative chemotherapy with fluorouracil and oxaliplatin versus fluorouracil alone in locally advanced rectal cancer: initial results of the German CAO/ARO/AIO-04 randomised phase 3 trial. Lancet Oncol. 2012;13(7):679-687.

8. Bedard PL, Hansen AR, Ratain MJ, Siu LL. Tumour heterogeneity in the clinic. Nature. 2013;501(7467):355.

9. Das P, Skibber JM, Rodriguez-Bigas MA, et al. Predictors of tumor response and downstaging in patients who receive preoperative chemoradiation for rectal cancer. Cancer. 2007;109(9):1750-1755.

10. Ryan JE, Warrier SK, Lynch AC, Ramsay RG, Phillips WA, Heriot AG. Predicting pathological complete response to neoadjuvant chemoradiotherapy in locally advanced rectal cancer: a systematic review. Colorectal Dis. 2016;18(3):234-246.

11. Ojima E, Inoue Y, Miki C, Mori M, Kusunoki M. Effectiveness of gene expression profiling for response prediction of rectal cancer to preoperative radiotherapy. $J$ Gastroenterol. 2007;42(9):730-736.

12. Watanabe T, Komuro Y, Kiyomatsu T, et al. Prediction of sensitivity of rectal cancer cells in response to preoperative radiotherapy by DNA microarray analysis of gene expression profiles. Cancer Res. 2006;66(7):3370.

13. Dresen RC, Beets GL, Rutten HJ, et al. Locally advanced rectal cancer: MR imaging for restaging after neoadjuvant radiation therapy with concomitant chemotherapy, Part I. Are we able to predict tumor confined to the rectal wall? Radiology. 2009;252(1):71-80.
14. Stanley K, Tait D, Chau I, Brown GJ. MRI predictive factors for tumor response in rectal cancer following neoadjuvant chemoradiation therapy-implications for induction chemotherapy? Int J Radiat Oncol Biol Phys. 2013;87(3):505-511.

15. Chang GJ, You YN, Park IJ, et al. Pre-treatment high-resolution rectal MRI and treatment response to neoadjuvant chemoradiation. Dis Colon Rectum. 2012;55(4):371.

16. Lambin P, Riosvelazquez E, Leijenaar R, et al. Radiomics: extracting more information from medical images using advanced feature analysis. Eur J Cancer. 2012;48(4):441-446.

17. Liu Z, Wang Y, Liu X, et al. Radiomics analysis allows for precise prediction of epilepsy in patients with low-grade gliomas. Neuroimage Clin. 2018;19:271-278.

18. Guo J, Liu Z, Shen C, et al. MR-based radiomics signature in differentiating ocular adnexal lymphoma from idiopathic orbital inflammation. Eur. Radiol. 2018;28(9):3872-3881.

19. Huang Y-Q, Liang C-H, He L, et al. Development and validation of a radiomics nomogram for preoperative prediction of lymph node metastasis in colorectal cancer. J. Clin. Oncol. 2016;34(18):2157-2164.

20. Shen $\mathrm{C}$, Liu Z, Wang Z, et al. Building CT radiomics based nomogram for preoperative esophageal cancer patients lymph node metastasis prediction. Transl Oncol. 2018;11(3):815-824.

21. Huang Y, Liu Z, He L, et al. Radiomics signature: a potential biomarker for the prediction of disease-free survival in earlystage (i or ii) non-small cell lung cancer. Radiology. 2016;281(3):947-957.

22. Zhang B, Tian J, Dong D, et al. Radiomics features of multiparametric MRI as novel prognostic factors in advanced nasopharyngeal carcinoma. Clin Cancer Res. 2017;23(15):4259-4269.

23. Liu Z, Zhang X-Y, Shi Y-J, et al. Radiomics analysis for evaluation of pathological complete response to neoadjuvant chemoradiotherapy in locally advanced rectal cancer. Clin Cancer Res. 2017;23(23):7253-7262.

24. Bulens P, Couwenberg A, Haustermans K, et al. Development and validation of an MRI-based model to predict response to chemoradiotherapy for rectal cancer. Radiother Oncol. 2018;126(3):437-442.

25. Tang Z, Zhang X-Y, Liu Z, et al. Quantitative analysis of diffusion weighted imaging to predict pathological good response to neoadjuvant chemoradiation for locally advanced rectal cancer. Radiother Oncol. 2019;132:100-108.

26. Intven M, Reerink O, Philippens ME. Dynamic contrast enhanced MR imaging for rectal cancer response assessment after neoadjuvant chemoradiation. $J$ Magn Reson Imaging. 2015;41(6): 1646-1653.

27. Intven M, Reerink O, Philippens M. Diffusion-weighted MRI in locally advanced rectal cancer. Strahlenther Onkol. 2013;189(2):117-122.

28. Engelen SM, Beets-Tan RG, Lahaye MJ, et al. MRI after chemoradiotherapy of rectal cancer: a useful tool to select patients for local excision. Dis Colon Rectum. 2010;53(7):979-986.

29. Janssen MH, Öllers MC, Riedl RG, et al. Accurate prediction of pathological rectal tumor response after two weeks of preoperative radiochemotherapy using $18 \mathrm{~F}$-fluorodeoxyglucose-positron emission tomography-computed tomography imaging. Int $J$ Radiat Oncol Biol Phys. 2010;77(2):392-399.

30. Nie K, Shi L, Chen Q, et al. Rectal cancer: assessment of neoadjuvant chemoradiation outcome based on radiomics of multiparametric MRI. Clin Cancer Res. 2016;22(21):5256-5264.

31. Park YA, Sohn SJ, Baik SH, Lee KY, Kim NK, Cho CW. Serum CEA as a predictor for the response to preoperative chemoradiation in rectal cancer. J Surg Oncol. 2010;93(2):145-150. 
32. Trakarnsanga A, Gönen M, Shia J, et al. Comparison of tumor regression grade systems for locally advanced rectal cancer after multimodality treatment. J Natl Cancer Inst. 2014;106(10): pii: dju248.

33. Kramer AA, Zimmerman JE. Assessing the calibration of mortality benchmarks in critical care: the Hosmer-Lemeshow test revisited. Crit Care Med. 2007;35(9):2052-2056.

34. Youden WJ. Index for rating diagnostic tests. Cancer. 1950;3(1):32-35.

35. Davnall F, Yip CSP, Ljungqvist G, et al. Assessment of tumor heterogeneity: an emerging imaging tool for clinical practice? Insights Imaging. 2012;3(6):573-589.

36. Ng F, Kozarski R, Ganeshan B, Goh V. Assessment of tumor heterogeneity by CT texture analysis: can the largest cross-sectional area be used as an alternative to whole tumor analysis? Eur J Radiol. 2013;82(2):342-348.

37. De Cecco CN, Ganeshan B, Ciolina M, et al. Texture analysis as imaging biomarker of tumoral response to neoadjuvant chemoradiotherapy in rectal cancer patients studied with 3-T magnetic resonance. Investig Radiol. 2015;50(4):239-245.
38. Dinapoli N, Barbaro B, Gatta R, et al. Magnetic resonance, vendor-independent, intensity histogram analysis predicting pathological complete response after radiochemotherapy of rectal cancer. Int J Radiat Oncol Biol Phys. 2018;102(4):765-774.

39. Huerta S, Gao X, Saha D. Mechanisms of resistance to ionizing radiation in rectal cancer. Expert Rev Mol Diagn. 2009;9(5):469.

40. Huerta $S$. Rectal cancer and importance of chemoradiation in the treatment. Adv Exp Med Biol. 2010;685(1):124-133.

41. Larue RT, Defraene G, De Ruysscher D, Lambin P, Van Elmpt W. Quantitative radiomics studies for tissue characterization: a review of technology and methodological procedures. $\mathrm{Br} \mathrm{J}$ Radiol. 2017;90(1070):20160665.

Publisher's Note Springer Nature remains neutral with regard to jurisdictional claims in published maps and institutional affiliations. 